\title{
Electron temperatures during rapid subauroral ion drift events
}

\author{
R. J. Moffett ${ }^{1}$, A. E. Ennis ${ }^{1}$, G. J. Bailey ${ }^{1}$, R. A. Heelis ${ }^{2}$, L. H. Brace ${ }^{3}$ \\ ${ }^{1}$ Upper Atmosphere Modelling Group, School of Mathematics and Statistics, University of Sheffield, Sheffield S3 7RH, UK \\ ${ }^{2}$ William B. Hanson Center for Space Sciences, University of Texas at Dallas, Richardson, TX 75083, USA \\ ${ }^{3}$ Space Physics Research Laboratory, University of Michigan, Ann Arbor, MI 48109-2143, USA
}

Received: 24 February 1997 / Revised: 29 October 1997 / Accepted: 20 November 1997

\begin{abstract}
Examples of data from DE-2 satellite instruments are presented. These illustrate the behaviour of plasma parameters in the F-region and adjacent topside ionosphere during rapid sub-auroral ion drift (SAID) events. In particular, a variety of behaviours of the electron temperature $\left(T_{e}\right)$ is demonstrated, both within and equatorward of the SAID region. The Sheffield University plasmasphere-ionosphere model (SUPIM) is used to perform calculations in which a model SAID is applied to a plasma flux tube. The model results indicate that strongly elevated ion temperature (a recognised signature of SAID events) is on occasion sufficient to raise $T_{e}$ to observed values by ion-electron heat transfer. On other occasions, an additional heat source is required. It is suggested that such a source for the electron gas may be due to interaction between the ring current and the plasmasphere at high altitudes. The magnitude of the downward heat flux is consistent with that necessary to produce sub-auroral red arcs. The resulting strongly heated electron gas causes vibrational excitation of molecular nitrogen in the thermosphere.
\end{abstract}

Key words. Electron temperature $\cdot$ Subauroral ion drift · Plasmasphere-ionosphere SUPIM

\section{Introduction}

Occurrences of a narrow band of rapid westward ion drift (of order $1 \mathrm{~km} \mathrm{~s}^{-1}$ ) in the evening sector of the subauroral ionosphere were detected by instruments on board satellites. References to an early observation (Galperin et al., 1973) and to subsequent clarifications of the properties and signatures of these drifts (known as

Correspondence to: R. J. Moffett

Fax: + 4411422 23739; e-mail: r.moffett@sheffield.ac.uk
SAID) may be found in the work of Anderson et al., (1993), who investigated the temporal relationship between SAID and auroral substorms. A phenomenological model of SAID production, based on the ideas of Southwood and Wolf (1978) and Harel et al. (1981), has been proposed by Anderson et al. (1993) in order to account for the observed ionospheric signatures and their temporal relationship with substorms.

Using the observed ion drifts as inputs, mathematical models of the ionosphere have been used to study the causes of the observed signatures of SAID (Sellek et al., 1991, 1992; Moffett et al., 1991, 1992a,b, 1993; Körösmezey et al., 1992; Heelis et al., 1993). Elevated and anisotropic ion temperatures, depletion of electron density, changes in ion composition, enhanced fieldaligned plasma flows and the behaviour of $\mathrm{H}^{+}$and $\mathrm{He}^{+}$ in the topside ionosphere have been modelled for various SAID conditions.

The behaviour of the electron temperature $\left(T_{e}\right)$ has been neglected, apart from a passing mention by Körösmezey et al. (1992). The chief reason for the neglect is the sparseness of published data of $T_{e}$ during SAID events. The present paper shows examples of observed $T_{e}$ (Sect. 2). Also, the Sheffield University plasmasphere-ionosphere model (SUPIM), with inclusion of ion-temperature anisotropy and vibrational excitation of molecular nitrogen (Sect. 3), is used to interpret the $T_{e}$ data (Sect. 4). It is found that ionelectron heat transfer due to the elevated ion temperature $\left(T_{i}\right)$ is sometimes sufficient to generate the $T_{e}$ values, but on other occasions an additional heat source for the electron gas is required, since $T_{e}$ may become comparable with $T_{i}$.

\section{Observations of SAID}

The Dynamics Explorer-2 satellite was launched into a $90^{\circ}$ inclination orbit in August, 1981, with apogee near $1000 \mathrm{~km}$ altitude and perigee near $300 \mathrm{~km}$ altitude. The DE-2 instruments giving data of interest in this study are the ion drift meter (Heelis et al., 1981), the retarding 
potential analyser (RPA) (Hanson et al., 1981) and the Langmuir probe (Krehbiel et al., 1981). The drift meter provided data on the bulk ion drift velocity in the plane perpendicular to the satellite track, nominally east-west and up-down. The RPA provided the ion concentration and composition and the ion temperature and bulk velocity in the direction of satellite motion. The Langmuir probe provided the electron concentration and temperature.

Figure 1 shows DE-2 data from a SAID encountered at about $420 \mathrm{~km}$ altitude on day 280 (7 October) in 1982. The top panel illustrates the characteristic spike in the east-west ion drift, with a maximum magnitude of about $2 \mathrm{~km} \mathrm{~s}^{-1}$ at 08:34:40 UT. The peak in $T_{i}$, trough in $N_{i}$ and peak in $T_{e}$ are collocated with the maximum drift. The $N_{e}$ measured by the Langmuir probe (not shown) agrees with the total ion concentration in the bottom panel.

The elevation of $T_{i}$ above its background value in the plasmasphere is caused by ion-neutral frictional heating (Schunk et al., 1976; Anderson et al., 1991; Sellek et al., 1991). St.-Maurice and Schunk (1977) predicted theoretically that the ion thermal velocity distribution becomes anisotropic and is distorted towards a toroidal form when the ion drift exceeds the neutral wind speed by more than the neutral thermal speed. Toroidal distributions had been observed by the RPA on the AE-C satellite (St.-Maurice et al., 1976). More recently, incoherent-scatter radar observations have given evidence for $T_{i}^{\perp}>T_{i}^{\|}$, where $T_{i}^{\perp}$ and $T_{i}^{\|}$are the perpendicular and parallel ion velocity distribution temperatures, respectively (Lockwood et al., 1993, and references therein). In the present context, the RPA essentially sees the perpendicular velocity distribution and thus measures $T_{i}^{\perp}$, which is expected to be significantly greater than $T_{i}^{\|}$(Moffett et al., 1993, and references therein). It is to be noted that the DE-2 satellite passes almost radially through the $N_{e}$ trough region where the ion drifts are aligned almost perpendicular to the meridian, and thus gradients in the ram ion drift are not expected to be large. Along the satellite track, the maximum density gradient is about one order of magnitude in about $12 \mathrm{~s}$. This amounts to less than a $10 \%$ change in the density over the nominal $0.5 \mathrm{~s}$ it takes to apply the retarding potential. The effects of this gradient on the derived temperature are small and less than the $8 \%$ error in the overall least-square analysis procedure.

The increase in the effective temperature in the loss rates for $\mathrm{O}^{+}$with $\mathrm{N}_{2}$ and $\mathrm{O}_{2}$ (St.-Maurice and Torr, 1978) leads to increased abundance of $\mathrm{NO}^{+}$and $\mathrm{O}_{2}^{+}$ relative to $\mathrm{O}^{+}$(Anderson et al., 1991; Moffett et al., 1992a) but a net decrease in $N_{e}$ in the F-region. The depth of the trough is emphasised by being embedded in the evening sector mid-latitude trough (Anderson et al., 1991), which may be substantially formed by plasma stagnation (Spiro et al., 1978).

The $T_{e}$ value reaches $4500 \mathrm{~K}$, comparable with $T_{i}$, during the SAID event. Since heat transfer from the ion gas to the electron gas is roughly proportional to temperature difference, this is prima facie evidence that

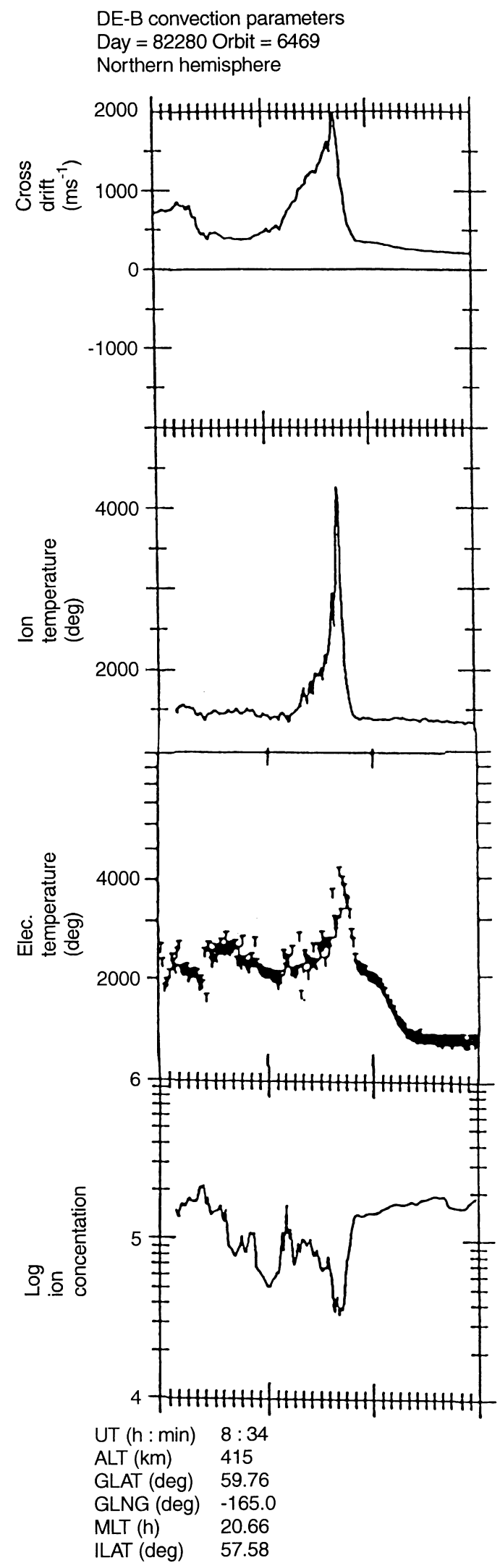

Fig. 1. Observations by DE-2 of a SAID event seen after 0834 UT on day 180, 1982. Top panel: zonal component of horizontal ion drift; second panel: ion temperature; third panel: electron temperature; bottom panel: total ion concentration 
the electrons have an additional heat source. The latitudinal profile of $T_{e}$ also shows a "shoulder" that is located equatorward of the SAID $T_{e}$ spike. This shoulder lies within the outer plasmasphere. There is experimental evidence that $T_{e}$ typically increases with increasing latitude in the outer plasmasphere on the nightside (Brace et al., 1982, 1988). The nightside heating could arise from photoelectron energy that is stored in the plasmasphere during the daytime (Brace et al., 1988) but may also necessitate another and stronger heat source.

In the second example of SAID data (Fig. 2), the ion drift velocity at about $320 \mathrm{~km}$ altitude reaches $4 \mathrm{~km} \mathrm{~s}^{-1}$ westward at about 0943 UT, with the ion temperature as measured by the RPA about $5000 \mathrm{~K}$. The $T_{e}$ profile shows four features worthy of note: (1) a shoulder from about 0941 to 0942UT: (2) an elevation to about $3000 \mathrm{~K}$, between 0942 and $0943 \mathrm{UT}$, followed by (3) a minimum; and (4) a spike reaching $2300 \mathrm{~K}$, where the ion drift speed is at its greatest value. Remarks on feature (1) are similar to those already made on the $T_{e}$ shoulder in Fig. 1. In this case, in addition, $N_{e}$ is decreasing with latitude and thus less heat (from whatever source) will be necessary to cause the shoulder. Features (2) and (3) indicate that additional heating of the electron gas may take place equatorward of the SAID or that the observed plasma may have been heated previously in a SAID region. On the other hand, feature (4) (the spike in $T_{e}$ in the SAID event) may be produced by ion-electron heat transfer alone, given the relatively large $T_{i}$ observed.

The final example (Fig. 3) shows another form of $T_{e}$ behaviour. The value of $T_{e}$ increases steadily with latitude in the outer plasmasphere but is still less than $2000 \mathrm{~K}$ before the SAID event is encountered (just after 0912 UT). A complication is the reducing solar zenith angle $\left(<100^{\circ}\right)$, leading to some direct solar heating of the electron gas. In this case, within the SAID region, $T_{e}$ rises towards $T_{i}$, as in Fig. 1.

\section{Plasmasphere-ionosphere model}

Bailey and Balan (1996) have described the standard version of SUPIM that is based on the model of Bailey and Sellek (1990) and now includes an eccentric dipole geomagnetic field. This version is used for low- and moderate-latitude applications. Briefly, values of the concentrations, field-aligned fluxes and temperatures of the $\mathrm{O}^{+}, \mathrm{H}^{+}, \mathrm{He}^{+}, \mathrm{N}_{2}^{+}, \mathrm{O}_{2}^{+}$and $\mathrm{NO}^{+}$ions, and the electrons, are obtained from time-dependent mathematical equations describing the chemical and physical processes controlling the behaviour of the thermal plasma. These equations, for the continuity, momentum and energy balance of the plasma confined within closed magnetic flux tubes, are solved along the axis of an eccentric dipole flux tube connecting the conjugate hemispheres.

For the present application, two major recent amendments to SUPIM have been utilised: (1) allowance for temperature anisotropy in $\mathrm{O}^{+}$and $\mathrm{NO}^{+}$, with

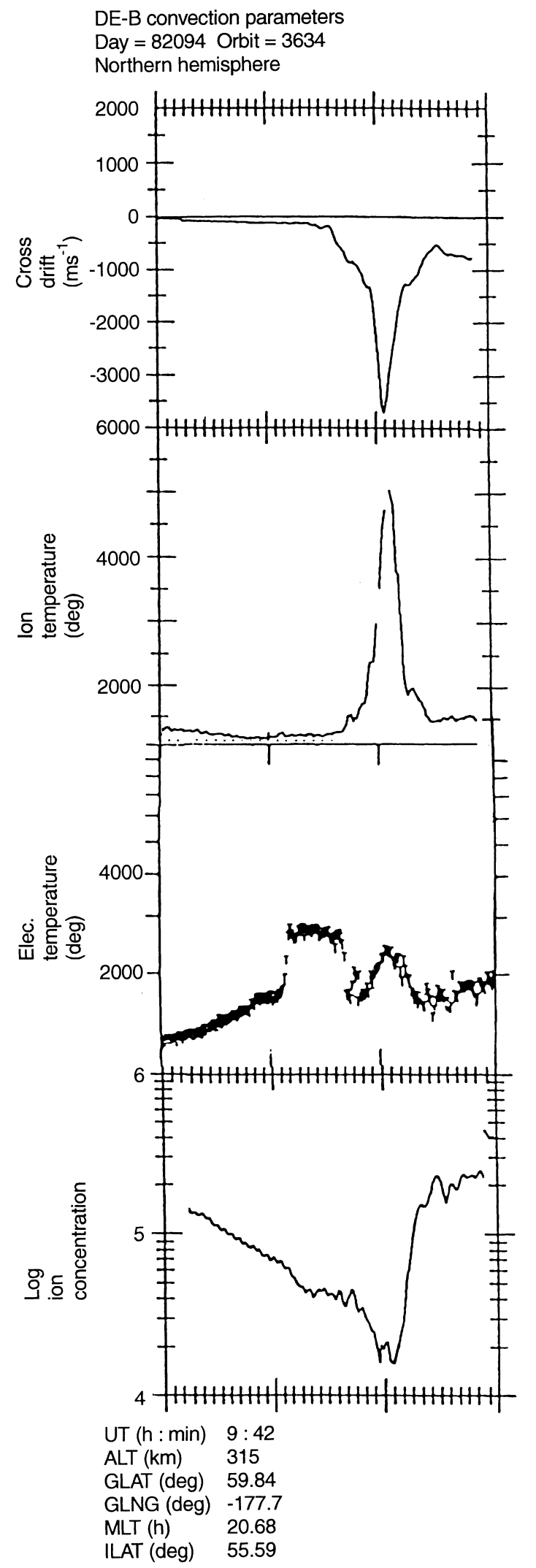

Fig. 2. SAID event observed after 0942 UT on day 94, 1982

these ions also having separately calculated temperatures (Moffett et al., 1993; Jenkins et al., 1997); (2) inclusion of vibrational excitation of molecular nitrogen 


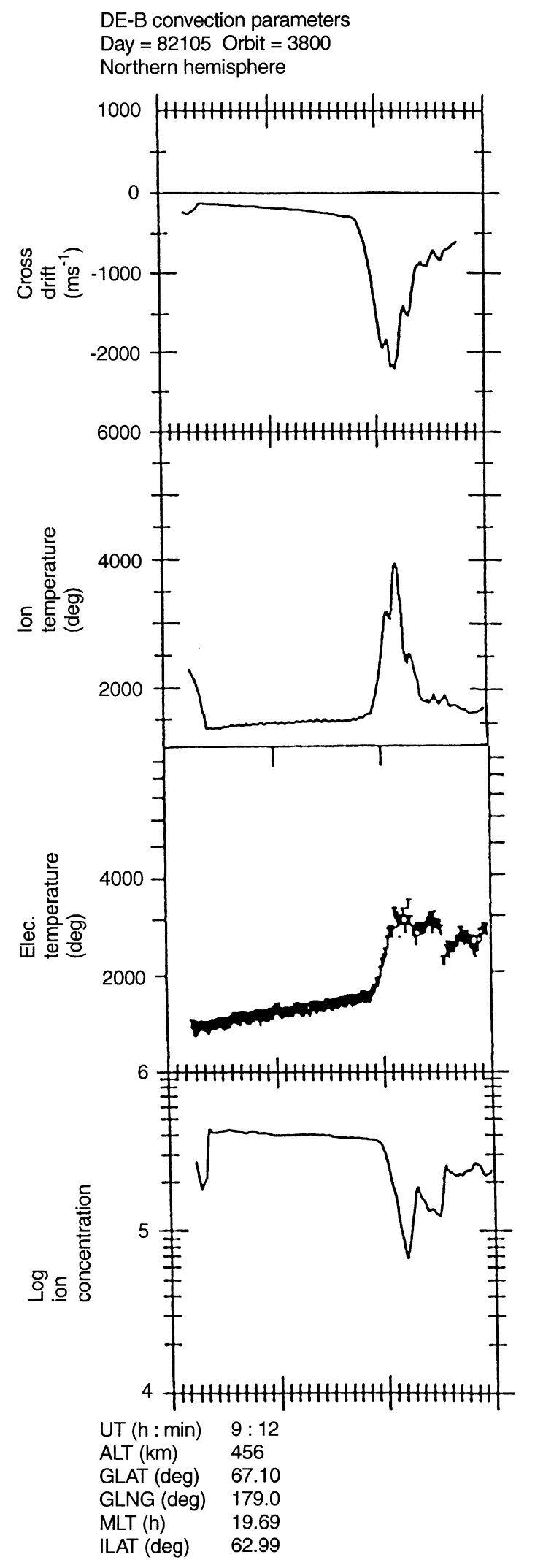

Fig. 3. SAID event observed after 0911 UT on day 105, 1982

(Ennis et al., 1995). In amendment (1), the ion-neutral frictional heating, $F_{i n}$, is partitioned into the parallel and perpendicular ion distributions according to ion fric- tional heating partition coefficients, $B_{\|}$and $B_{\perp}$. Thus $B_{\|} F_{\text {in }}$ is input to the parallel ion energy balance equation and $B_{\perp} F_{\text {in }}$ to the perpendicular ion energy balance equation. The values of $B_{\|}$and $B_{\perp}$ are based on the Monte Carlo cross-sections of Winkler et al. (1992), whose results were expressed in terms of temperature coefficients, $\beta_{\|}$and $\beta_{\perp}$. The coefficients $B_{\|}$and $B_{\perp}$ depend on the composition of the ion and neutral gases. A more detailed explanation is given by Moffett et al. (1993), in which it is pointed out that expressing $T_{\|}$and $T_{\perp}$ in terms of $\beta_{\|}$and $\beta_{\perp}$ is valid only in the region where the frictional heating is intense and the ion density relatively high (whereas the $B_{\|} F_{i n}$ and $B_{\perp} F_{\text {in }}$ terms can be used at all altitudes). Typical values for $B_{\|}$and $B_{\perp}$ have been presented by Jenkins et al. (1997). For amendment (2), the inclusion of vibrationally excited $\mathrm{N}_{2}$ (denoted by $\mathrm{N}_{2}^{*}$ ), the continuity and diffusion equations for the first five vibrational states of $\mathrm{N}_{2}$ have been solved. The main source of $\mathrm{N}_{2}^{*}$ is excitation by the high-energy tail of the energy distribution of the thermal electron gas. The main loss process of $\mathrm{N}_{2}^{*}$, at the altitudes of interest, is through collisions with atomic oxygen.

\section{Model runs and results}

The model has been used to investigate the processes that may have to be invoked to explain the data. Model results shown here are for 1982 equinox (day 82), an $L$ value of $4, F 10.7=150 \times 10^{-22} \mathrm{~W} \mathrm{~m}^{-2} \mathrm{~Hz}^{-1}$ and $A_{p}=4$. The MSIS-86 neutral model atmosphere (Hedin, 1987) gives the neutral concentrations and temperatures; the neutral air wind velocities are taken from the HWM 90 thermospheric wind model (Hedin et al., 1991); and the solar EUV fluxes from the EUV 91 solar EUV flux model (Tobiska, 1991).

The model was run for 32 model hours, with a corotating flux tube starting at local noon and finishing at $2000 \mathrm{LT}$. The neutral atmosphere conditions were then held at those relevant to $2000 \mathrm{LT}$. In order to see the undisturbed evolution of the model ionosphere, a 90min continuation run was carried out with only the corotation velocity and no applied SAID. Starting from the same initial conditions, the undisturbed evolution was followed for $15 \mathrm{~min}$, after which time a sudden westward $\mathbf{E} \times \mathbf{B}$ drift of $2 \mathrm{~km} \mathrm{~s}^{-1}$ was applied for a period of $30 \mathrm{~min}$, so that comparison can be made with the data of Fig. 1. The drift was then turned off and the subsequent behaviour followed. The time after a continuation run is started is called elapsed time. A further set of calculations was made with the use of an additional high-altitude heat source for the electron gas. The heat source was applied linearly about the equator, switched on at the same time as the applied SAID (i.e. at $15 \mathrm{~min}$ elapsed time) and lasted for $20 \mathrm{~min}$. In all cases, the model calculations were repeated with the inclusion of vibrationally excited nitrogen.

It should be pointed out that many studies (for example, Spiro et al., 1978; Quegan et al., 1982; Anderson et al., 1993) suggest that a depleted density 
in the mid-latitude trough region can be explained by the presence of slow or stagnant flow that is moving poleward in darkness. Thus the background density level, upon which the SAID and/or the high-altitude heat source must act, may be lower than that used in these calculations; we simply have assumed that the background plasma density profile used to define the trough is taken to be constant and equal to the values in the auroral zone poleward of the event and in the corotating plasmasphere equatorward of the event.

The results displayed in Figs. 4-8 illustrate the response of the model ionosphere to the input drift magnitude and atmospheric conditions appropriate to the SAID event of Fig. 1. The ion temperature profiles of Fig. 4 show the elevated ion temperatures, compared with the unperturbed temperature, which are a characteristic signature of the SAID event. The $\mathrm{O}^{+}$gas has a greater temperature anisotropy than the $\mathrm{NO}^{+}$gas because the frictional heat due to the ion-neutral relative motion is partitioned more strongly into the perpendicular distribution for the $\mathrm{O}^{+}$compared to the $\mathrm{NO}^{+}$. The curve labelled $T_{i}^{\prime}$ shows the composition-weighted average of the three-dimensional temperatures for the $\mathrm{O}^{+}$ and $\mathrm{NO}^{+}$, i.e.,

$$
\begin{aligned}
& T_{i}^{\prime}=\left\{N\left(\mathrm{O}^{+}\right)\left(\frac{1}{3} T_{\|}\left(\mathrm{O}^{+}\right)+\frac{2}{3} T_{\perp}\left(\mathrm{O}^{+}\right)\right)\right. \\
& \left.+N\left(\mathrm{NO}^{+}\right)\left(\frac{1}{3} T_{\|}\left(\mathrm{NO}^{+}\right)+\frac{2}{3} T_{\perp}\left(\mathrm{NO}^{+}\right)\right)\right\} /\left\{N\left(\mathrm{O}^{+}\right)+N\left(\mathrm{NO}^{+}\right)\right\},
\end{aligned}
$$

where $N\left(\mathrm{O}^{+}\right), N\left(\mathrm{NO}^{+}\right)$are the $\mathrm{O}^{+}$and $\mathrm{NO}^{+}$concentrations, respectively, $T_{\|}\left(\mathrm{O}^{+}\right)$and $T_{\|}\left(\mathrm{NO}^{+}\right)$are the parallel temperatures of the ion gases and $T_{\perp}\left(\mathrm{O}^{+}\right)$and $T_{\perp}\left(\mathrm{NO}^{+}\right)$ the perpendicular temperatures; here $\mathrm{O}_{2}^{+}$has been neglected, since it appears to play a minor role in the

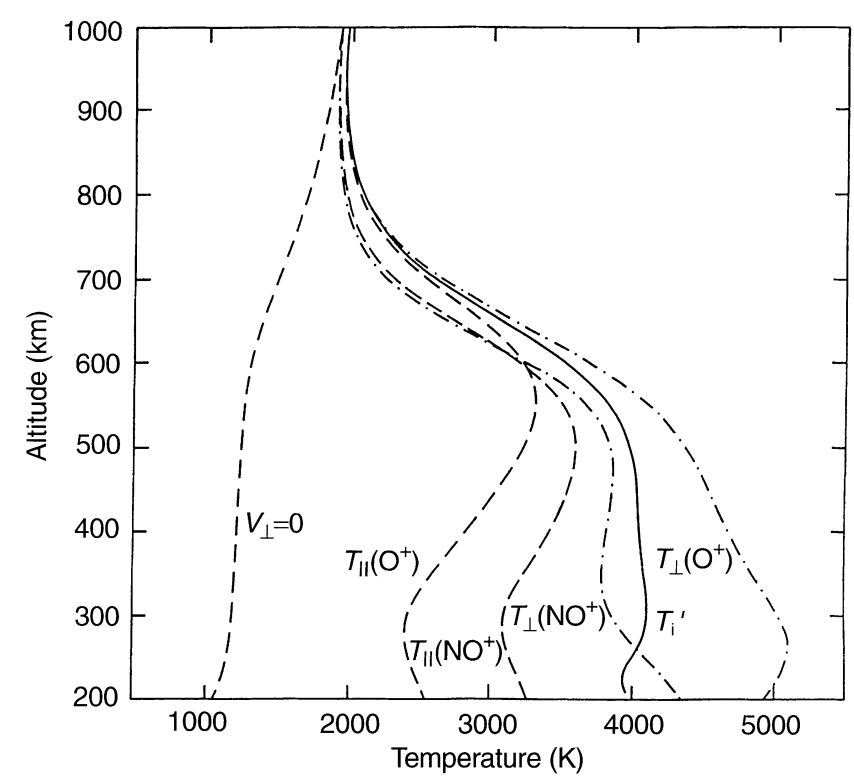

Fig. 4. Model ion temperature profiles at 30 min elapsed time for a drift of $2 \mathrm{~km} \mathrm{~s}^{-1}$ for atmospheric conditions appropriate to Fig. 1. Shown are parallel and perpendicular $\mathrm{O}^{+}$and $\mathrm{NO}^{+}$temperatures; $T_{i}^{\prime}$ is the composition-weighted three-dimensional average [see Eq. (1)] molecular ion abundance during SAID events (Moffett et al., 1992a). This average ion temperature enters into the electron-ion heat transfer term in the heat balance equations.

The following factors determine the behaviour of the ion temperatures. Frictional heating is the dominant heat input below about $350 \mathrm{~km}$ altitude and this is countered by collisional cooling to the neutral gas. In the topside ionosphere the initial temperature increase is dominated by field-aligned advective transport of hot plasma from lower altitudes until the plasma attains a new scale height (Heelis et al., 1993). The time evolution of the ion temperature is determined by the relatively small contributions of heat conduction in the parallel and perpendicular ion distributions, and by collisional heat transfer between the ion gases and between the ions and electrons.

This time evolution is illustrated in Fig. 5 for $420 \mathrm{~km}$ altitude, corresponding to the observations of Fig. 1. The average ion temperature, $T_{i}^{\prime}$, is shown because, as already noted, this is the ion temperature that enters into the electron-ion collisional heat transfer term in the heat balance equations. The other ion temperature, that of the $\mathrm{O}^{+}$perpendicular distribution, dominates the temperature measured by the RPA. The satellite is moving almost perpendicular to the magnetic field and, at $420 \mathrm{~km}$ altitude, $\mathrm{NO}^{+}$contributes only a few percent to the ion abundance. The agreement of the model $T_{\perp}\left(\mathrm{O}^{+}\right)$and the observed quantity (Fig. 1) is satisfactory.

Also presented in Fig. 5 are the $T_{e}$ and $N_{e}$ values during the model SAID event. The electron temperature rises quickly in response to heat transfer from the ion gas at elevated temperature. During the remainder of the event, $T_{e}$ remains almost constant at around $200 \mathrm{~K}$.
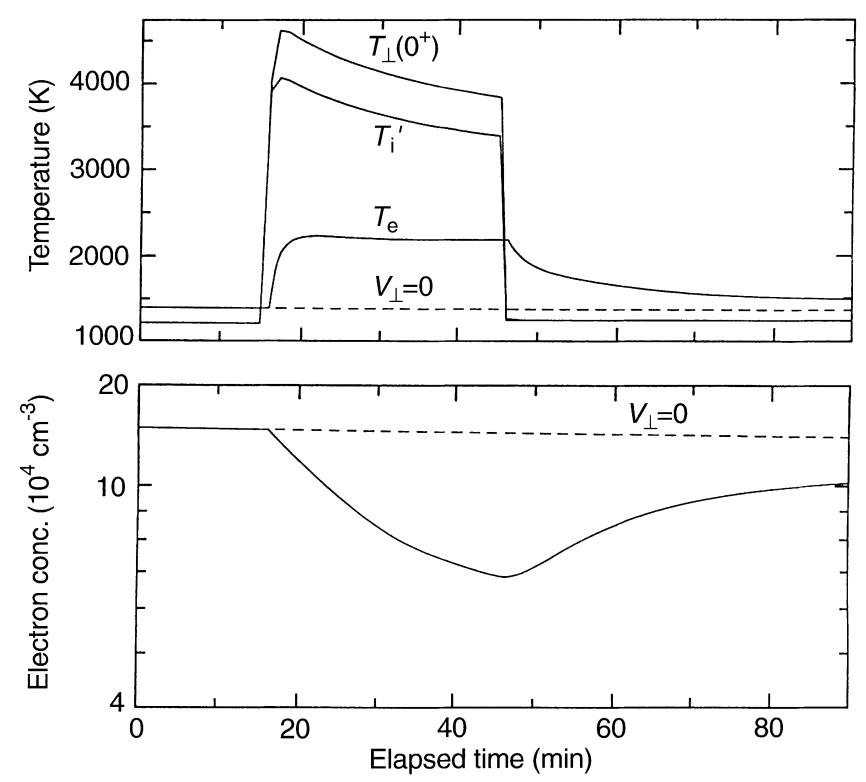

Fig. 5. Model ion temperatures, electron temperature and electron concentration, for an altitude of $420 \mathrm{~km}$, as a function of elapsed time for the same inputs as those of Fig. 4 (without an additional heat source at high altitudes) 
Examination of the heat balance terms for the electron gas (results not shown) reveals that this value of $T_{e}$ and those of the F-region represent a balance between ionelectron and electron-neutral collisional heat transfers. The decay in the electron density is caused by increased loss rate of $\mathrm{O}^{+}$due to increased effective temperature for $\mathrm{O}^{+}-\mathrm{N}_{2}$ and $\mathrm{O}^{+}-\mathrm{O}_{2}$ collisions.

The results in Figs. 4 and 5 were obtained without allowance for vibrational excitation of $\mathrm{N}_{2}$. When the runs were repeated with the inclusion of vibrationally excited $\mathrm{N}_{2}$, the results (not shown here) differed very little. This is readily explained by noting that the $T_{e}$ values of Fig. 5 are not high enough to give significant amounts of $\mathrm{N}_{2}^{*}$; it was found that the abundance of $\mathrm{N}_{2}^{*}$ was less than $5 \%$ at $420 \mathrm{~km}$ altitude at 45 min elapsed time. Comparison of Figs. 1 and 5 shows clearly that, while the model results cannot be expected to mimic the observational results exactly, the disagreement in the maximum value of $T_{e}$ is severe.

The effects on $T_{e}$ of the uncertainty in the calculated values of $N_{e}$ can be assessed both qualitatively and quantitatively. If the initial values of $N_{e}$ are increased, the modelled trough values will be far from agreement with the observed values. If the initial values of $N_{e}$ are decreased, ion-electron heat transfer will be affected more (since it is proportional to $N_{e}^{2}$ ) than electronneutral heat transfer or electron thermal conduction. Thus the need for an additional heat source would be strengthened. The magnitude of the proposed heat source depends of the local value of $N_{e}$ and could be lower than that used in our calculations. These qualitative assessments were confirmed by computations that used differing initial $N_{e}$ and so gave differing $N_{e}$ results within the SAID event. Detailed results are not present- ed. To quote one example, however: for results corresponding to Fig. 5, when the $N_{e}$ minimum is lowered to $4 \times 10^{4} \mathrm{~cm}^{-3}, T_{e}$ reaches $2000 \mathrm{~K}$ within the SAID event.

The obvious candidate to improve the model results is an additional heat source for the electron gas due to ring current-plasmasphere interaction (Kozyra et al., 1987; Chandler et al., 1988; Fok et al., 1991). Heat produced at great altitudes is conducted downwards by the electron gas to the ionosphere, raising the electron temperature and thus sometimes causing SAR arcs. The heat flux to the ionosphere in the absence of the additional heat source is shown in Fig. 6. It is seen that at $1000 \mathrm{~km}$ altitude the downward flux is about $10^{9} \mathrm{eV} \mathrm{cm}^{-2} \mathrm{~s}^{-1}$. It is found that about an order of magnitude greater flux is required to elevate $T_{e}$ to the observed level. Such a flux is consistent with the studies by Rees and Roble (1975) and Kozyra et al. $(1987 ; 1990)$ on SAR arcs. For the results presented in Figs. 6-8, the equatorial heat source was turned on at the time of onset of the model SAID (15 min elapsed time) and switched off at 36 min elapsed time. Figure 6 also shows the boosted downward heat flux.

The effects of this flux are demonstrated in Fig. 7 (without $\mathrm{N}_{2}^{*}$ ) and Fig. 8 (with $\mathrm{N}_{2}^{*}$ ). In both cases, the average ion temperature, $T_{i}^{\prime}$, and the $\mathrm{O}^{+}$perpendicular temperature show little change from their values in Fig. 5, for $420 \mathrm{~km}$ altitude. In the F-region and adjacent topside, frictional heating and ion-neutral heat transfer dominate the ion thermal balance. Larger changes are evident, however, in the topside ionosphere (above about $500 \mathrm{~km}$; results not shown) since ion-electron heat transfer contributes to the ion heat balance there.

By contrast, $T_{e}$ responds significantly to the increased electron heat flux, both directly and indirectly. In Fig. 7

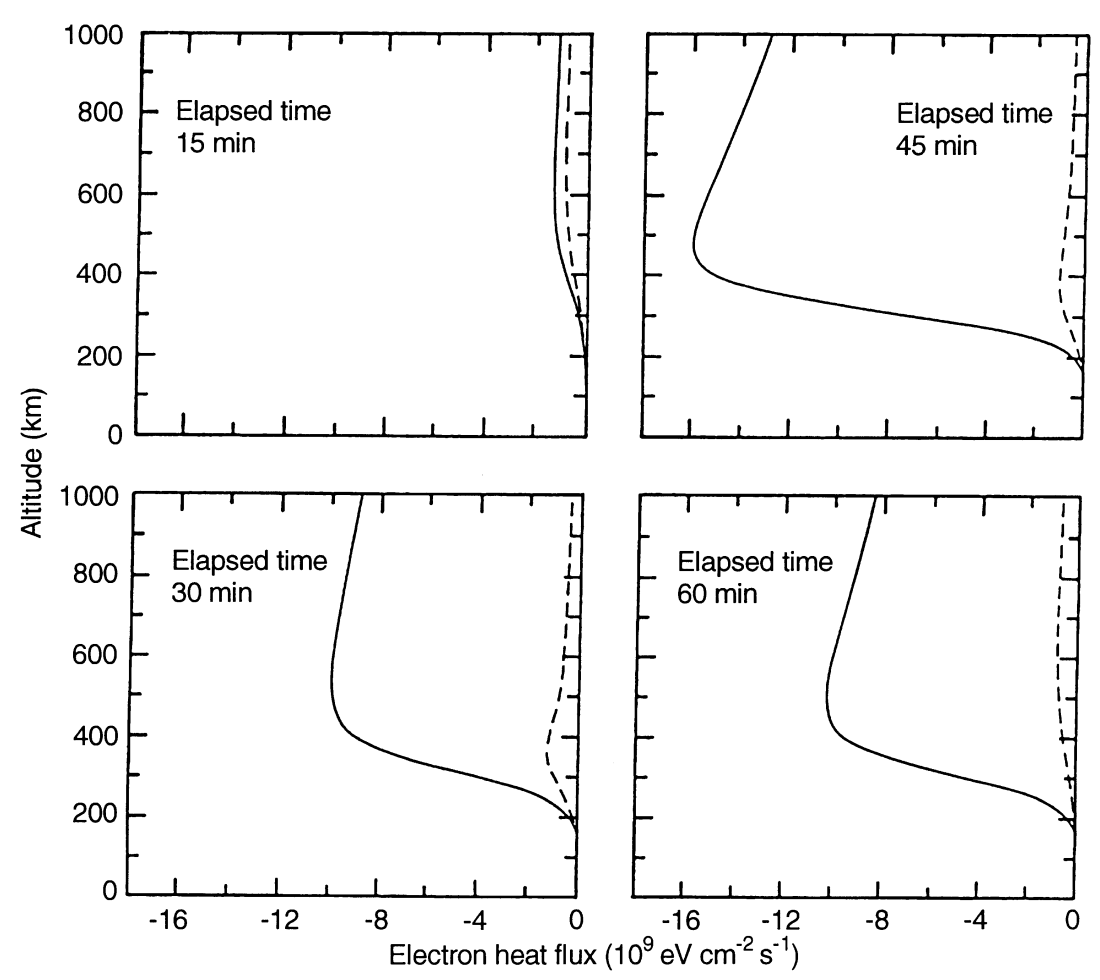

Fig. 6. Model profiles of heat flux in the electron gas: without additional heat source (dashed line) with additional heat source (solid line) 

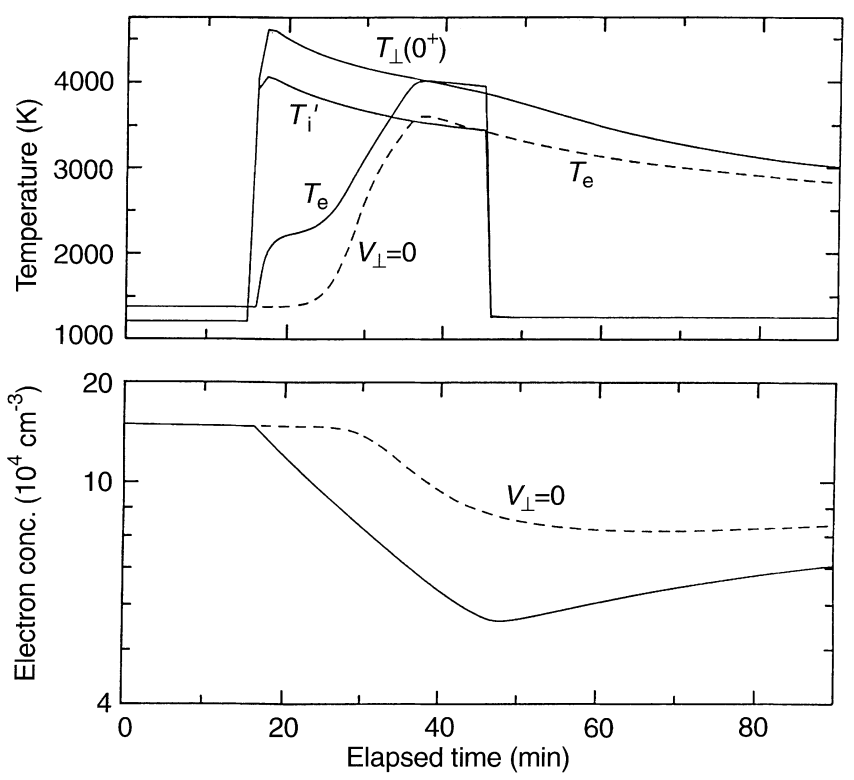

Fig. 7. As for Fig. 5, but with inclusion of additional heat source for the electron gas

(without $\mathrm{N}_{2}^{*}$ ) $T_{e}$ peaks at $4000 \mathrm{~K}$; the strong convergence of the heat flux as the $T_{e}$ altitude gradient changes leads to deposit of heat in the electron gas. The further reduction of the $N_{e}$ value in the trough (Fig. 7) relative to that in Fig. 5 is due to a change of shape of the $N_{e}$ altitude profile (results not shown); the level of the F2peak is lowered by the increase in the plasma temperature $\left(T_{e}+T_{i}\right)$. Vibrationally excited $\mathrm{N}_{2}$ gives a greater loss rate with $\mathrm{O}^{+}$than unexcited $\mathrm{N}_{2}$, leading to reduced $\mathrm{N}_{e}$, which decreases the heat capacity of the electron gas, tending to raise $T_{e}$ again. Thus the effects of inclusion of $\mathrm{N}_{2}^{*}$ (Fig. 8) are to lower the $N_{e}$ minimum to $5 \times 10^{4} \mathrm{~cm}^{-3}$ and to raise $T_{e}$ further, to a maximum of
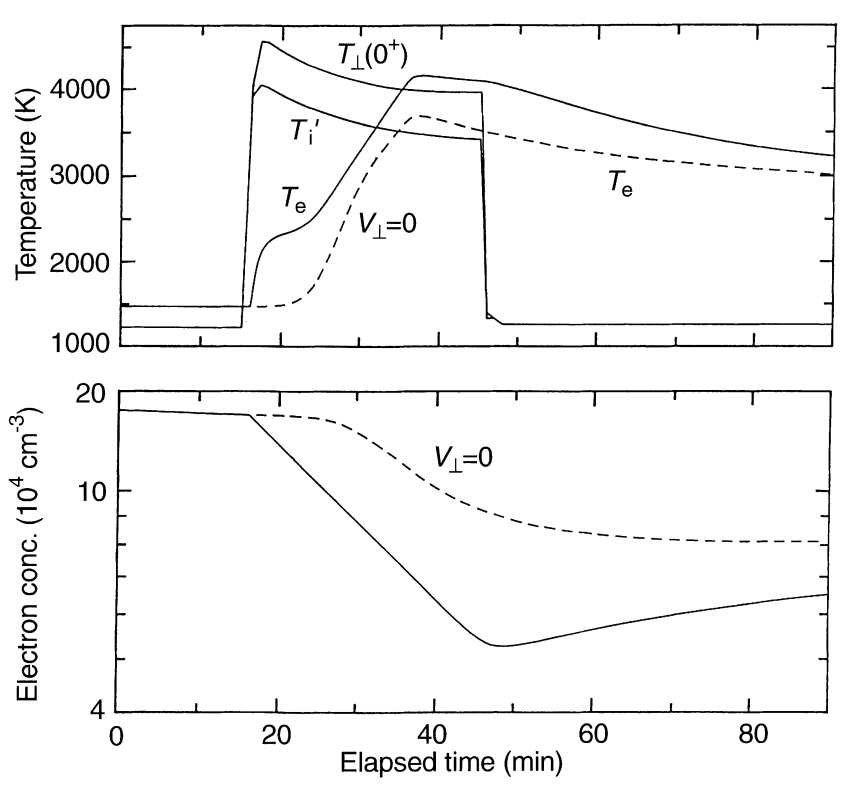

Fig. 8. As for Fig. 7, but with inclusion of vibrationally excited $\mathrm{N}_{2}$
$4200 \mathrm{~K}$. For the results of Fig. 8, the abundance of $\mathrm{N}_{2}^{*}$ was about $10 \%$ (at 45 min elapsed time at $420 \mathrm{~km}$ ).

Control results in Figs. 5, 7 and 8 (i.e. with no SAID) also reveal the effects of the additional heat source for the electrons. Figure 5 simply shows that, with neither the additional heat source nor the frictional heat source, $T_{e}$ remains unperturbed. Figure 8 shows that the additional heat source, with the help of $\mathrm{N}_{2}^{*}$, can alone raise $T_{e}$ to about $3800 \mathrm{~K}$.

The behaviour of $T_{e}$ around 20 min elapsed time in Figs. 7 and 8 may be tentatively suggested as relevant to the spatial shoulders in the observed $T_{e}$ (Figs. 1 and 3) already mentioned. With $N_{e}$ still relatively high, the effect of the downward heat flux shows a tendency to flatten out, whereas at later times, as the $N_{e}$ trough deepens, the $T_{e}$ value climbs to about $4000 \mathrm{~K}$. Further investigation is needed to determine whether a further contribution to the increasing $T_{e}$ within the shoulder is due to energy stored at high altitudes in the plasmasphere.

Finally, for this set of model results related to Fig. 1, Fig. 9 illustrates the dependence of the $T_{e}$ and $N_{e}$ results on the temporal phasing of the additional heat input. If the additional heat input is moved forward by $15 \mathrm{~min}$ (now starting at 0 min elapsed time and ending at 21 min elapsed time, while the SAID still begins at 15 min elapsed time), $T_{e}$ reaches a plateau at about $3750 \mathrm{~K}$. It is noticeable in Figs. 7-9 that the time required for cooling of the electron gas after the SAID event is significantly greater than that required for heating the gas. About $45 \mathrm{~min}$ after the event, $T_{e}$ has decreased to about $3000 \mathrm{~K}$.

Computations have also been performed with a SAID $V_{\perp}$ of $4 \mathrm{~km} \mathrm{~s}^{-1}$. In this case the high ion-neutral relative velocity causes high ion temperatures (Fig. 10), leading to increased abundances of $\mathrm{NO}^{+}$. This is consistent with experimental data and model results
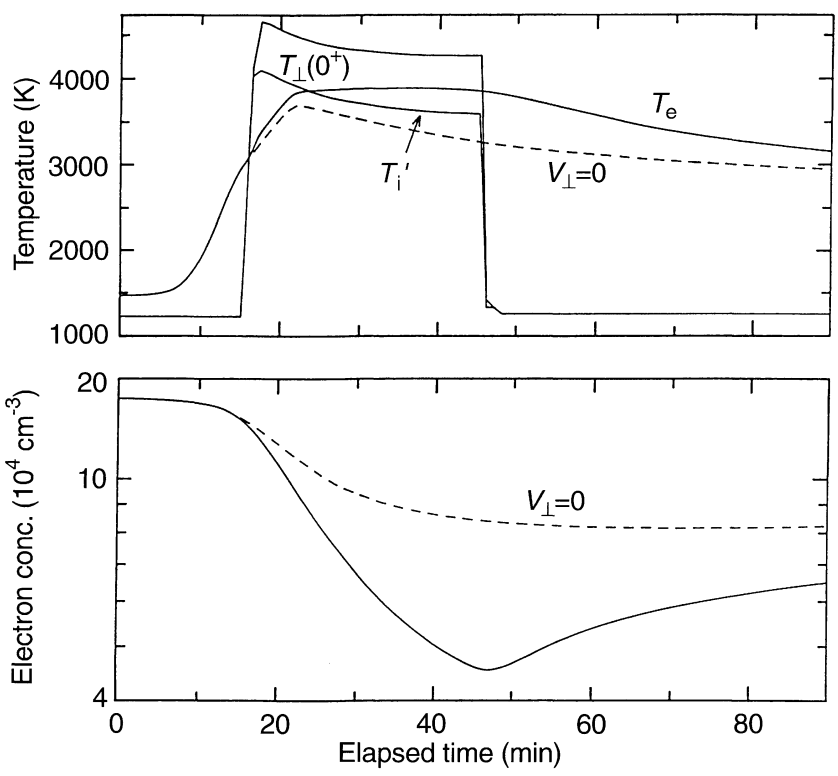

Fig. 9. As for Fig. 8, but with shift in timing of additional heat source (see text) 

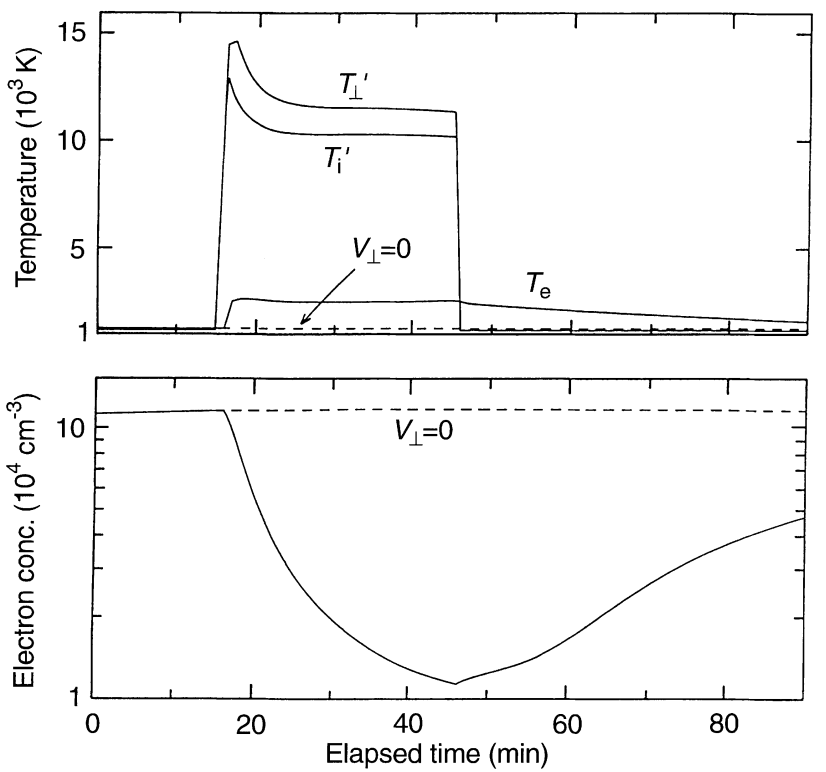

Fig. 10. Model ion temperatures, electron temperature and electron concentration, for an altitude of $320 \mathrm{~km}$, as a function of elapsed time for input drift of $4 \mathrm{~km} \mathrm{~s}^{-1}$ (without additional heat source and $\mathrm{N}_{2}^{*}$ )

presented previously (Anderson et al., 1991; Moffett et al., 1992a). In Fig. 10 is shown the compositionweighted average perpendicular ion temperature, denoted by $T_{\perp}^{\prime}$, for $320 \mathrm{~km}$ altitude. This is the parameter most likely to be comparable with the ion temperature measured by the RPA instrument (Fig. 2). Also shown in Fig. 10 are $T_{i}^{\prime}$ and $T_{e}$. The former, defined by Eq. (1), is the composition-weighted average three-dimensional ion temperature. The difference $\left(T_{i}^{\prime}-T_{e}\right)$ enters into the rate of collisional heat transfer between ions and electrons. It is striking that, although this difference is very much greater in the $4-\mathrm{km} \mathrm{s}^{-1}$ case compared to the $2-\mathrm{km} \mathrm{s}^{-1}$ case (Fig. 5), the resulting $T_{e}$ is only slightly greater. It is readily shown that the reduction in heat transfer from ions to electrons is due to the severe reduction in $N_{e}$ (Fig. 10); ion-electron heat transfer depends on $N_{e}^{2}$, whereas electron-neutral heat transfer depends on $N_{e}$ and the neutral concentration.

Results in Fig. 11 show the effects of including an additional heat input and vibrationally excited nitrogen. The heat input was chosen as the same at that used in the $2-\mathrm{km} \mathrm{s}^{-1} V_{\perp}$ case, so that the downward fluxes of heat in the electron gas are similar to those of Fig. 6. As in Fig. $8, T_{e}$ exceeds $4000 \mathrm{~K}$ by the end of the event. The $N_{e}$ value is only slightly affected.

\section{Discussion}

Examples of observed electron temperature behaviour in SAID events have been presented. These demonstrate responses of $T_{e}$ to particular SAID conditions. Model simulations of SAID events have been performed in order to try to identify the main physical processes that lead to the observed $T_{e}$ behaviour.

However, when discussing the issues that arise from the model-observational comparisons, two general ca-
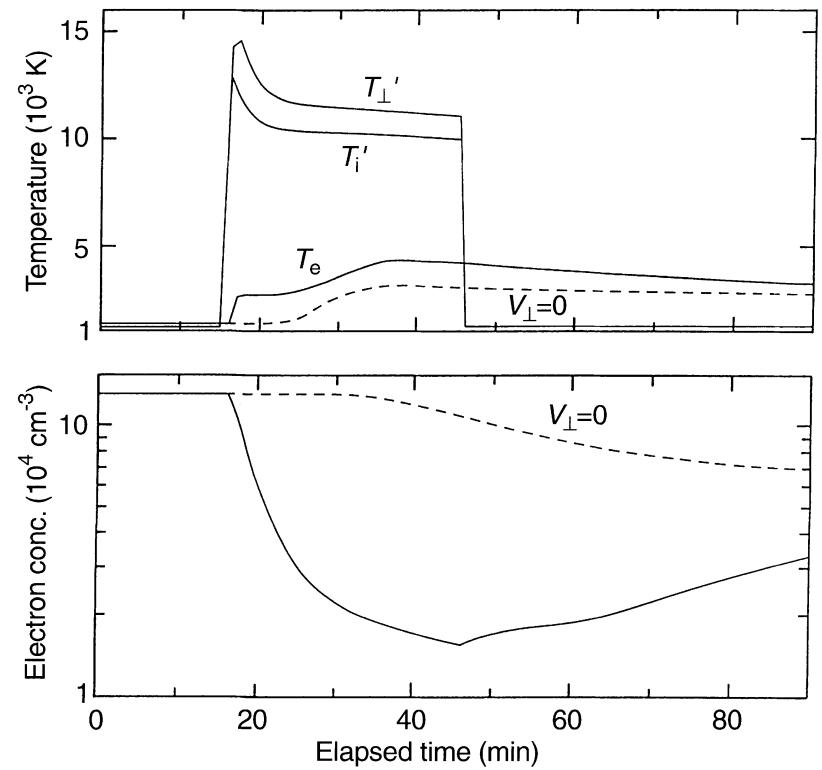

Fig. 11. As for Fig. 10, but with additional heat source and inclusion of $\mathrm{N}_{2}^{*}$

veats must be borne in mind. First, the observations have been obtained along the single trajectories of the satellite. Each set of results represents a snapshot of a SAID event over a period of a few minutes. In contrast, the model calculations are performed for an $\mathbf{E} \times \mathbf{B}$ drifting tube of plasma. Care must therefore be exercised in attempting to separate spatial or temporal features. As a flux tube enters the SAID region, the temporal behaviour of the plasma is simulated by the model calculations; the spatial structure at a certain time is made up of the total set of flux tubes entrained in the SAID event at that time. Thus the behaviour of the plasma within a single flux tube is indicative of the behaviour that is likely to be observed, but an exact correspondence is not possible without detailed information on the time evolution and spatial extent of the SAID. Secondly, the model $N_{e}$ values within the SAID event depend on the values of $N_{e}$ previous to the event. These initial values are not well known. The period of entrainment of the plasma in the convection stagnation region, prior to the SAID event, is also not known. Thus computations have been performed with differing initial values of $N_{e}$; these results confirm the discussion and conclusions that follow.

The modelled elevation and anisotropy of $T_{i}$ due to the SAID is in accord with the present and previous observational results and with previous model results. The resulting difference in $T_{i}$ and $T_{e}$ leads to heat transfer from the ions to the electrons. The results show that this can raise $T_{e}$ (from less than $1500 \mathrm{~K}$ ) to between 2000 and $3000 \mathrm{~K}$ for both the $V_{\perp}=2$ and $V_{\perp}=4 \mathrm{~km} \mathrm{~s}^{-1}$ cases; in the latter case, $N_{e}$ is likely to be less than in the former case and so compensates (in the rate of heat transfer) for the greater $T_{i}-T_{e}$ difference. This modest increase in $T_{e}$ is (1) clearly compatible with the results within the SAID region of Fig. 2, and (2) perhaps compatible with the SAID results of Fig. 3. 
It is evident also that the greater elevation of $T_{e}$ in the SAID region in Fig. 1 requires an additional heat source. The model results demonstrate that the downwards flux of heat usually invoked to explain the occurrence of SAR-arcs (Kozyra et al., 1987) is of the correct order of magnitude to explain the elevated $T_{e}$. Furthermore, Caton et al. (1996) found that downward heat flow from the magnetosphere was needed as an input in order to obtain agreement with EISCAT topside measurements.

Liu et al. (1995) and Caton et al. (1996) have also invoked the effects of soft electron precipitation in modelling electron temperature and plasma upflows observed by DE-2 and by EISCAT, respectively. By contrast, the SAID events lie equatorward of the region of electron precipitation that has a source in the plasma sheet. Mechanisms for producing the electric field in the trough may require the SAID to be embedded in the region of weak ion precipitation associated with the inner edge of the ring current. Thus any heat source required to account for elevated electron temperatures in SAID events is identified as arising from a source in the ring current.

The persistence of elevated $T_{e}$ after the SAID event in the model results bears on consideration of the elevated $T_{e}$ feature seen equatorward of the SAID in Fig. 2. This feature may arise directly from heating of the electron gas that is occurring at that location and that presumably arises from the ring current. However, the plasma with elevated $T_{e}$ may have recently emerged from a SAID region, and so the heating may have ceased. With the $T_{e}$ value about $3000 \mathrm{~K}$ in the feature, it is likely that the origin of the heated plasma still requires additional heating either in, or equatorward of, a SAID region.

Allowance for the vibrational excitation of molecular nitrogen by thermal electrons has negligible effect when the increase in $T_{e}$ is modest. This is to be expected, since the high-energy tail of the electron distribution is insufficiently intense. On the other hand, when the additional heat source is applied and $T_{e}$ is further elevated, vibrationally excited $\mathrm{N}_{2}$ plays a noticeable role. The excitation that occurs is sufficient to increase the loss rate of $\mathrm{O}^{+}$with $\mathrm{N}_{2}$ and so deepen the density trough within the SAID event. There is some positive feedback since lower $N_{e}$ leads to higher $T_{e}$.

\section{Conclusions}

From comparison of the observational and model results, it is concluded that:

1. The ion temperatures observed during SAID events are caused by ion-neutral frictional heating which is preferentially partitioned into the ion perpendicular distribution.

2. Heat transfer from the ion gas to the thermal electron gas can raise the electron temperature to between 2000 and $3000 \mathrm{~K}$.

3. To obtain greater elevations of electron temperature, an additional heat source for the electron gas is required. It is suggested that the likely candidate is ring current-plasmasphere interaction; the downward flux heat required in the present study is consistent with that used in modelling SAR arcs and in modelling topside measurements by EISCAT. The persistence of increased electron temperatures after a SAID event raises the possibility that plasma with elevated $T_{e}$ observed equatorward of a SAID may recently have emerged from such an event.

4. Vibrational excitation of molecular nitrogen by higher-energy thermal electrons can deepen the plasma density trough, associated with the SAID, by increasing the $\mathrm{O}^{+}$loss rate. This in turn allows the electron temperature to increase further.

Acknowledgements. This work was supported by grant GR/L 20238 from the Particle Physics and Astronomy Research Council at the University of Sheffield and by NSF grants ATM-9311392 and ATM-9615064 at the University of Texas at Dallas.

Topical Editor D. Alcaydé thanks J. L. Horwitz and J. Sojka for their help in evaluating this paper.

\section{References}

Anderson, P. C., W. B. Hanson, and R. A. Heelis, The ionospheric signatures of rapid subauroral ion drifts, J. Geophys. Res., 96, 5785, 1991.

Anderson, P. C., W. B. Hanson, R. A. Heelis, J. D. Craven, D. N. Baker, and L. A. Frank, A proposed production model of rapid subauroral ion drifts and their relationship to substorm evolution, J. Geophys. Res., 98, 6069, 1993.

Bailey, G. J., and N. Balan, A low-latitude ionosphere-plasmasphere model, STEP Handbook on Ionospheric Models, Ed. R. W. Schunk, Utah State University, p. 173; 1996.

Bailey, G. J., and R. Sellek, A mathematical model of the Earth's plasmasphere and its application in a study of $\mathrm{He}^{+}$at $L=3.0$, Ann. Geophysicae, 8, 171, 1990.

Brace, L. H., R. F. Theis, and W. R. Hoegy, A global view of Fregion electron density and temperature at solar maximum, Geophys. Res. Lett., 9, 989, 1982.

Brace, L. H., C. R. Chappell, M. O. Chandler, R. H. Comfort, J. L. Horwitz, and W. R. Hoegy, F-region electron temperature signatures of the plasmasphere based on Dynamics Explorer 1 and 2 measurements, J. Geophys. Res., 93, 1896, 1988.

Caton, R., J. L. Horwitz, P. G. Richards, and C. Liu, Modeling of F-region ionospheric upflows observed by EISCAT, Geophys. Res., Lett., 23, 1537, 1996.

Chandler, M. O., J. U. Kozyra, J. L. Horwitz, R. H. Comfort, and L. H. Brace, Modeling of the thermal plasma in the outer plasmasphere - a magnetospheric heat source, in Modeling magnetospheric plasma Eds. T. E. Moore and J. H. Waite Jr. AGU Monogr. 44, p. 101, 1988.

Ennis, A. E., G. J. Bailey, and R. J. Moffett, Vibrational nitrogen concentration in the ionosphere and its dependence on season and solar cycle, Ann. Geophysicae, 13, 1164, 1995.

Fok, M.-C., J. U. Kozyra, and L. H. Brace, Solar cycle variation in the subauroral electron temperature enhancement: comparison of AE-C and DE2 satellite observations, J. Geophys. Res., 96, 1861, 1991.

Galperin, Y. I., Y. N. Ponomarov, and A. G. Zosinova, Direct measurements of ion drift velocity in the upper atmosphere during a magnetic storm, Kosm. Issled., 11, 273, 1973.

Hanson, W. B., R. A. Heelis, R. A. Power, C. R. Lippincott, D. R. Zucarro, B. J. Holt, L. H. Harmon, and S. Sanatani, The retarding potential analyzer for Dynamics Explorer-B, Space Sci. Instrum., 5, 503, 1981 . 
Harel, M., R. A. Wolf, R. W. Spiro, P. H. Reiff, C. K. Chen, W. J. Burke, F. J. Rich, and M. Smiddy, Quantitative simulation of a magnetospheric substorm, 1, Comparison with observations, $J$. Geophys. Res., 86, 2261, 1981.

Hedin, A. E., MSIS-86 thermospheric model, J. Geophys. Res., 92, 4649, 1987.

Hedin, A. E., M. A. Biondi, R. G. Burnside, G. Hernandez, R. M. Johnson, T. L. Killeen, C. Mazaudier, J. W. Meriwether, J.E. Salah, R. J. Sica, R. W. Smith, N. W. Spencer, V. B. Wickwar, and T. S. Virdi, Revised global model of thermosphere winds using satellite and ground-based observations, J. Geophys. Res., 96, 7657, 1991.

Heelis, R. A., W. B. Hanson, C. R. Lippincott, D. R. Zucarro, L. H. Harmon, B. J. Holt, J. E. Doherty, and R. A. Power, The ion drift meter for Dynamics Explorer-B, Space Sci. Instrum., 5, 511,1981 .

Heelis, R. A., G. J. Bailey, R. Sellek, R. J. Moffett, and B. Jenkins, Field-aligned drifts in subauroral ion drift events, J. Geophys. Res., 98, 21493, 1993.

Jenkins, B., R. J. Moffett, J. A. Davies, and M. Lester, Nightside ion frictional heating: atomic and molecular ion temperature anisotropy and ion composition changes, J. Atmos. Solar-Terr. Phys., 59, 1329, 1997.

Körösmezey, A., C. E. Rasmussen, T. I. Gombosi, and G. V. Khazanov, Anisotropic ion heating and parallel $\mathrm{O}^{+}$acceleration in regions of rapid $\mathbf{E} \times \mathbf{B}$ convection, Geophys. Res. Lett., 19, 2289, 1992.

Kozyra, J. U., E. G. Shelley, R. H. Comfort, L. H. Brace, T. E. Cravens, and A. F. Nagy, The role of ring current $\mathrm{O}^{+}$in the formation of stable auroral red arcs, J. Geophys. Res., 92, 7487, 1987.

Kozyra, J. U., C. E. Valladares, H. C. Carlson, M. J. Buonsanto, and D. W. Slater, A theoretical study of the seasonal and solar cycle variations of stable auroral red arcs, J. Geophys. Res., 95, 12219, 1990.

Krehbiel, J. P., L. H. Brace, R. F. Theis, W. H. Pinkus, and R. B. Kaplan, The Dynamics Explorer Langmuir probe instrument, Space Sci. Instrum., 5, 493, 1981.

Liu, C., J. L. Horwitz, and P. G. Richards, Effects of frictional ion heating and soft-electron precipitation on high-latitude Fregion upflows, Geophys. Res. Lett., 22, 2713, 1995.

Lockwood, M., I. W. McCrea, G. H. Millward, R. J. Moffett, and H. Rishbeth, EISCAT observations of ion composition and temperature anisotropy in the high-latitude F-region, J. Atmos. Terr. Phys., 55, 895, 1993.

Moffett, R. J., R. Sellek, and G. J. Bailey, The behaviour of $\mathrm{H}^{+}$ and $\mathrm{He}^{+}$ions in the topside ionosphere during a short-lived subauroral ion drift, Planet. Space Sci., 39, 1209, 1991.
Moffett, R. J., R. A. Heelis, R. Sellek, and G. J. Bailey, The temporal evolution of the ionospheric signatures of subauroral ion drifts. Planet. Space Sci., 40, 663, 1992a.

Moffett, R. J., G. J. Bailey, and B. Jenkins, Effects of greatly increased $\mathrm{O}^{+}$loss in the ionospheric F-region, Planet. Space Sci., 40, 1631, 1992b.

Moffet, R. J., B. Jenkins, and G. J. Bailey, A modelling study of anisotropic ion temperatures generated in the F-layer by subauroral ion drifts. Ann. Geophysicae, 11, 1051, 1993.

Quegan, S., G. J. Bailey, R. J. Moffett, R. A. Heelis, T. J. FullerRowell, D. Rees, and R. W. Spiro, A theoretical study of the distribution of ionization in the high-latitude ionosphere and plasmasphere: first results on the mid-latitude trough and the light-ion trough, J. Atmos. Terr. Phys., 44, 619, 1982.

Rees, M. H., and R. G. Roble, Observations and theory of the formation of stable auroral red arcs, Rev. Geophys. Space Phys., 13, 201, 1975.

Schunk, R. W., P. M. Banks, and W. J. Raitt, Effects of electric fields and other processes upon the nighttime high-latitude $\mathrm{F}$ layer, J. Geophys. Res., 81, 3271, 1976.

Sellek, R., G. J. Bailey, R. J. Moffett, R. A. Heelis, and P. C. Anderson, Effects of large zonal plasma drifts on the subauroral ionosphere, J. Atmos. Terr. Phys., 53, 557, 1991.

Sellek, R., R. J. Moffett, and G. J. Bailey, Effects of rapid zonal plasma flow in the mid-latitude ionosphere: modulation of the field-aligned plasma flow by neutral air upwelling, $A d v$. Space Res., 12, (6), 171, 1992.

Southwood, D. J., and R. A. Wolf, An assessment of the role of precipitation in magnetospheric convection, J. Geophys. Res., 83, 5227, 1978.

Spiro, R. W., R. A. Heelis, and W. B. Hanson, Ion convection and the formation of the mid-latitude F-region ionization trough, J. Geophys. Res., 83, 4255, 1978.

St.-Maurice, J.-P., and R. W. Schunk, Auroral ion velocity distributions for a polarization collision model, Planet. Space Sci., 25, 243, 1977.

St.-Maurice, J.-P., and D. G. Torr, Nonthermal rate coefficients in the ionosphere: the reactions of $\mathrm{O}^{+}$with $\mathrm{N}_{2}, \mathrm{O}_{2}$ and $\mathrm{NO}$, J. Geophys. Res., 83, 969, 1978.

St.-Maurice, J.-P., W. B. Hanson, and J. C. G. Walker, Retarding potential analyzer measurements of the effect of ion-neutral collisions on the ion velocity distribution in the auroral ionosphere, J. Geophys. Res., 81, 5438, 1976.

Tobiska, W. K., Revised solar extreme ultraviolet flux model, J. Atmos. Terr. Phys., 53, 1005, 1991.

Winkler, E., J.-P. St.-Maurice, and A. R. Barakat, Results from improved Monte Carlo calculations of auroral ion velocity distributions, J. Geophys. Res., 97, 8399, 1992. 